\title{
Posterior capsulorhexis combined with optic buttonholing: an alternative to standard in-the-bag implantation of sharp-edged intraocular lenses? A critical analysis of 1000 consecutive cases
}

\author{
R. Menapace
}

Received: 25 December 2007 / Revised: 20 January 2008 /Accepted: 24 January 2008 / Published online: 19 April 2008

(C) The Author(s) 2008

\begin{abstract}
Background Current after-cataract prevention relies on optimizing the natural barrier effect of the optic rim against lens epithelial cell (LEC) migration. However, deficiencies in circumferential capsular bag closure caused by the intraocular lens (IOL) haptic or delayed secondary re-division of the fused capsules by Soemmering's ring formation lead to primary or secondary barrier failure. Consequently, surprisingly high posterior laser capsulotomy rates have been reported long-term, even with optimal capsular surgery and the most widespread hydrophobic acrylic IOLs, considered to be the most advanced. Intraoperative removal of the central posterior capsule has been shown to be effective in further reducing LEC immigration. However, efficacy has turned out to be limited because of the propensity of LECs to use the posterior optic surface as an alternative scaffold.

Material and methods

- Technique: in pediatric cataract surgery, buttoning-in of the optic into an adequately-centered posterior capsulorhexis opening has been described previously. This technique was further elaborated and applied as the standard technique in a large series of adult eyes. In general, the diameter aimed at was $5-6 \mathrm{~mm}$ for the anterior, and 4-5 $\mathrm{mm}$ for the posterior capsulorhexis. Between September 2004 and June 2007, 1000 consec-
\end{abstract}

The author has no proprietary interest in any of the materials or equipment mentioned in this paper.

R. Menapace $(\bowtie)$

Department of Ophthalmology,

University of Vienna Medical School,

Waehringer Guertel 18-20,

Vienna 1090, Austria

e-mail: rupert.menapace@meduniwien.ac.at utive cases have thus been performed and systematically evaluated. One hundred and fifty eyes additionally underwent extensive anterior LEC abrasion. Another sub-series investigated the option of further reducing capsular fibrosis by creating an anterior capsulorhexis larger than the optic.

- Evaluation: special scrutiny was applied to detect postoperative vitreous entrapment. Regeneratory and fibrotic after-cataract formation were both meticulously followed-up. Postoperative pressure course, anterior segment inflammation, macular thickness and morphology, as well as axial optic stability and optic centration, were evaluated in intraindividual comparison studies.

Results A low rate of vitreous complications was found, which can be avoided by appropriate surgery. Vitreous entanglement occurred in six eyes, and vitreous herniation after PPCCC over-sizing in two. In three, anterior vitrectomy was performed. There was only one single case of retinal detachment - supposedly unrelated to the technique itself - and no case of cystoid macular edema. Retro-optical regenerate formation was completely abolished, while fibrosis was drastically reduced by the posterior capsule sandwiched in between the anterior LEC layer on the backside of the anterior capsule and the anterior optic surface, thereby blocking contact-mediated myofibroblastic LEC transdifferentiation. Additional capsular polishing further reduced residual fibrosis emerging from the anterior capsule contacting the optic adjacent to the haptic junction, as well as regeneratory LEC re-proliferation on the posterior capsule overlying the optic. Postoperative pressure course was almost identical to that found after standard in-the-bag implantation of the IOL, as was flare, and macular thickness and morphology. As opposed to bagfixated IOLs, no axial movement of the optic was detected. 
IOL optics always perfectly centered even when the capsular opening was not optimally centered. Due to the exquisite stretchability and elasticity of the posterior capsule, the 6-mm IOL optic could safely be buttoned-in in a posterior capsulorhexis of $4 \mathrm{~mm}$ and smaller.

Conclusions Posterior optic buttonholing (POBH) is a safe and effective technique which not only excludes retro-optical opacification, but also withholds capsular fibrosis by obviating direct contact between the anterior capsular leaf and the optic surface. Anterior LEC abrasion significantly reduced both the residual fibrosis and regeneratory LEC proliferation. Apart from pediatric cataract, $\mathrm{POBH}$ is currently recommended for eyes with pseudoexfoliation syndrome, high axial myopia, peripheral retinal disease, and multifocal IOL implantation. Toric IOLs and magnet-driven accommodative IOL systems are other potential applications. Generally, POBH holds promise for becoming a routine alternative to standard in-the-bag IOL implantation in the future.

Keywords Posterior capsule opacification · Fibrosis .

Regeneratory after-cataract $\cdot$ Primary posterior capsulorhexis · Reclosure - Optic buttonholing . Vitreous entrapment · IOP · Flare · Cystoid macular edema . Retinal detachment - Axial optic shift - Optic centration . Toric IOLs

\section{Background}

The current concept of after-cataract prevention ("posterior capsule opacification", or "PCO") is based on fully exploiting the natural barrier effect of the optic rim against lens epithelial cell (LEC) migration. This barrier effect is inherent to any optic rim, even when rounded. However, it can be optimized by the following measures: (1) by providing a circumferential overlap of the optic by the anterior capsular leaf, (2) by sharpening the posterior optic edge, and (3) by enhancing the collagenous sealing of both capsular leaves along the optic rim [10]. The latter is achieved by using fibrosis-inducing optic materials, and by preserving the integrity of the LEC layer as the substrate of fibrosis. The importance of fibrotic sealing is mirrored by two facts: (1) intraocular lenses (IOLs) made of materials with a lower fibrogenetic potential, such as acrylics, undergo a sharp increase in after-cataract score after 3-5 years [27], while with those made from materials with a higher fibrogenetic potential, such as silicones, it remains fairly constant, and (2) LEC abrasion by anterior capsule polishing significantly increases regeneratory after-cataract formation and the need for Nd: YAG laser capsulotomy (YAG-LCT) [11]. Implementation of all three above-mentioned parameters into modern cataract surgery has led to a drastical reduction of after-cataract and YAG-LCT rates.
However, utilization of the edge barrier effect has two major shortcomings: (1) overlap of the optic by the anterior capsule leaf induces fibrotic whitening and shrinkage of the latter, thereby reducing the size of the free optic zone (this is especially true if the rhexis opening is small or decentered, be it because of inadequate dimensioning or due to subsequent contraction), and (2) the barrier function can be lacking from the very beginning, or wear off over the years, even when all the three above-mentioned requirements are fully met.

The barrier cannot build up when fusion of both capsular leaves and consecutive bending of the posterior capsule at the posterior optic edge is not initiated. Such "primary barrier failure" can be limited to sectors or comprise the whole circumference of the optic. By nature, capsular fusion is impeded at the haptic-optic junction. While with looped haptics the barrier at the junction may remain fully preserved, broad-based haptics, including those used with the currently popular one-piece IOLs, inevitably disrupt the barrier at the optic insertion [24]. As a result, a gateway is opened up for LECs migrating from the germinative equatorial zone inwards. Deficiencies of capsular bag fusion may also arise independent of the haptic junction. When the reason is not evident, these have been termed "idiopathic primary barrier failure". Most often, however, it is caused by ovalization of the capsular bag by overly long and rigid haptics, which impedes capsular fusion along the longitudinal IOL axis.

Even when primary capsular fusion has been complete, the barrier may eventually fail. As Soemmering's ring formation sets in, the two capsular leaves are increasingly redivided from the capsular equator inwards. If collagenous sealing of the capsule leaves along the optic rim is insufficient, these are increasingly forced apart, and the bend at the posterior optic edge is finally annihilated. Dormant LECs may thus become reactivated [15] and access the retrolental space. This phenomenon has been termed "secondary barrier failure".

These barrier failures are the reason why in the long run YAG-LCT is still required in a considerable proportion of cases, even with optimized IOL designs and appropriate surgery. For the Acrysof 3-piece IOL, the reported YAGLCT rate at 5 years ranges between 16\% [2] and 27\% [own data in publication]. After 10 years, a cumulative YAGLCT rate of $42 \%$ was found (L. Vock, R. Menapace R. Long-term YAG laser capsulotomy and after-cataract rates with the sharp edge Acrysof and round edge PhacoFlex intraocular lenses: 10 year results. Abstract XXVth Congress of the ESCRS, 8.-12.9.2007, Stockholm). Thereby it must be kept in mind that the YAG-LCT rate only reflects those cases where central pearl formation is significant enough to interfere with vision. When also considering those eyes where LEC invasion remains limited to the 
periphery of the retrooptical capsule, the rate of barrier failure is significantly higher.

Therefore, the potential for avoiding after-cataract formation by optimizing the edge barrier effect is limited, even when all relevant parameters are fully exploited. In addition, even in those cases where the sharp edge forms a permanent circumferential barrier, visibility of the peripheral retina may be significantly reduced by pearl formation in the intercapsular space outside of the optic rim.

The sharp edge as such also has the severe disadvantage of immanently causing dysphotopsia. Negative dysphotopsia is especially annoying for the patient. In the consultation section of the April 2005 issue of the Journal of Cataract and Refractive Surgery, dysphotopsia has been classified the "problem number one of modern cataract surgery" [16]. Interestingly, this problem is not limited to acrylic IOLs with double square-edged (truncated) optics, but is also experienced with frosted edges, and even with rounded anterior edges. Also, it has been found with both acrylics and silicones [3].

\section{Rationale}

The still significant percentage of after-cataract formation requiring YAG-LCT in the longer run, and the problem of dysphotopsia, have fostered the search for better alternatives. Thereby, a new approach was required, since the concept of optimizing the migrational barrier effect of the optic edge is largely exploited.

As one option, intraoperative removal of the central posterior capsule by performing a primary posterior continuous curvilinear capsulorhexis (PPCCC) seemed promising, since the posterior capsule serves as a scaffold for centrally migrating LECs. As such, it is not an alternative, but much more supplements the current sharp edge concept by providing a "second barrier of defence" when the optic edge barrier is overcome by migrating LECs.
However, even though it has been shown to be safe [26, 4], the efficacy of adding a PPCCC is also limited. Firstly, the residual peripheral capsule can still be invaded and opacified by LECs which reduces the free optic zone. Secondly, similar to the anterior LEC ongrowth inside the ACCC rim often observed with acrylics [1], equatorial LECs can also grow over the PPCCC rim unto the free posterior optic surface, as they may utilize the latter as an alternative scaffold. Incidence and severity vary depending upon surface tension, wetability, and contact angle of the optic material, as well as the individual biological responsiveness, and is more pronounced in eyes with a defective blood-aqueous barrier [25]. We performed a randomized prospective intraindividual comparison study including 29 patients with bilateral PPCCC, whereby one eye received a silicone, the other a hydrophilic acrylic IOL. Since roundedged optic IOLs were used at that time, this made the full impact of a PPCCC more obvious. After 2 years, partial reclosure of the PPCCC opening due to peripheral LEC ongrowth was found in $55 \%$ of the acrylic, and in $28 \%$ of the silicone IOL eyes. Total reclosure of the PCCC was observed in two eyes of the acrylic and in one eye of the silicone IOL group [5] (Fig. 1). When evaluated with an objective grading system, after-cataract formation within the PPCCC opening exceeded 2 and 3 out of 10 possible grades for the silicone and acrylic IOL respectively.

Thus, a PPCCC constitutes a useful additive surgical measure, but does not fully prevent after-cataract formation.

One promising alternative concept is combining a PPCCC with posterior buttoning-in of the optic ("posterior optic buttonholing", or $\mathrm{POBH}$ ), a technique which has been reserved for pediatric cataract surgery [6]. By doing so, the posterior capsule ends up lying on top of the optic. Instead of hindering the LECs from migrating, these are diverted towards the anterior optic surface, thus bypassing the optic edge. By doing so, migrating LECs, by principle, can no longer access the retrolental space.

In order to elucidate the suitability of the posterior optic buttonholing $(\mathrm{POBH})$ technique as a routine procedure for
Fig. 1 Primary posterior capsulorhexis decreases YAG laser capsulotomy rate by removing scaffold for LEC migration; however, residual capsule may still opacify, and LECs may alternatively grow upon optic surface
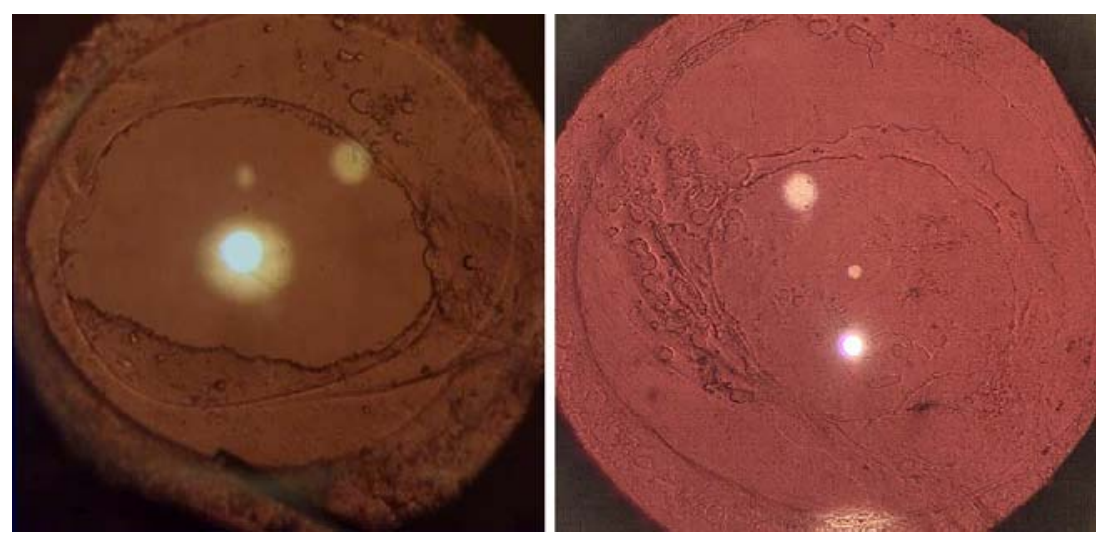
senile cataracts, an extensive prospective study comprising a large number of eyes was initiated. In several substudies, a prospective randomized bilateral design was used to elucidate the risk profile compared to the standard in-thebag implantation of IOLs in detail.

For this purpose, the surgical technique of POBH was revised and optimized to be then systematically investigated. From September 2004 to June 2007, over 1000 eyes underwent this $\mathrm{POBH}$ procedure. Patients were randomly taken from the waiting list. All prospective comparison studies were approved by the Medical University of Vienna Ethics Committee.

\section{Technique}

The surgical technique comprises an additional step of sophisticated, but perfectly controlled capsular and viscosurgery. After performing a standard 5-6 $\mathrm{mm}$ anterior continuous curvilinear capsulorhexis (ACCC), and after removing the lens contents with phacoemulsification and meticulously cleaning the capsular bag, the posterior capsule is flattened and the capsular fornix collapsed by pushing the iris and anterior capsule backwards with a low-viscosity cohesive ocular viscoelastic device (OVD, preferably Healon $\left.{ }^{\circledR}\right)$. Thereby, the pupil is additionally widened. Due to this pupil-dilating effect, all eyes with a pupil size larger than $4 \mathrm{~mm}$ under full pharmacological dilatation were amenable to the procedure. After incising the center of the posterior capsule with a 30-gauge hypodermic needle (Sterican ${ }^{\circledR}$ manufactured by B. Braun, Melsungen, or Fine-Ject ${ }^{\circledR}$ by Henke-Sass-Wolf, Tuttlingen, Germany), a small amount of OVD is injected through the capsular opening. Then, the edge of the incised capsule is taken up by Utrata forceps and the incision extended peripherally to create a well-centered $4-5 \mathrm{~mm}$ PPCCC opening. When after the first pass one quadrant of the PPCCC has been completed, OVD is again injected in order to separate the underlying anterior hyaloid surface from the posterior capsule. After completion of the PPCCC and removal of the flap, OVD is again injected, this time in order to ensure full circumferential separation of the anterior hyaloid membrane from the residual posterior capsule up to the very periphery. Adequate viscoseparation of capsule and hyaloid is considered crucial to allow safe buttoning-in of the optic, and will be addressed in more detail in the Discussion Section below. Then, an open-loop IOL is implanted through an injector and fixated in the capsular bag fornix. When both loops are placed in the bag, the optic is buttoned-in into the PPCCC opening by gently pressing it down. As the elastic posterior capsule thereby wraps around the optic periphery in between the haptic junctions, the distended capsule and the optic form a mechanically stable and watertight diaphragm (Figs. 2 and $3)$. Finally, the OVD is aspirated from the anterior chamber, the globe is tonisized, and the paracenteses are hydrated. The cataract incision is left unsutured. The surgery is performed under topical plus optional intracameral anesthesia (Lidocaine $4 \%$ plus Lidocaine unpreserved $1 \%$ ) to ensure bright and stable retroillumination during the PPCCC manoeuvre. The HOYA AF-1 with a 6$\mathrm{mm}$ optic is preferably used due to its particular haptic design (continuous optic-haptic transition: Fig. 4; "hopper loop"). Should the PPCCC result to be larger than the optic, as may occur in eyes with lax zonules or a large capsular bag, a $6.5-\mathrm{mm}$ optic IOL should be resorted to, in order to ensure sealing of the diaphragm by sufficient circumferential capsular overlap. Additional implantation of a capsular tension ring facilitates estimation and performance of an appropriately sized and centered PPCCC in these eyes [9].

Approximately 150 eyes underwent additional anterior capsule polishing with a special LEC aspiration curette [13] (Fig. 5), the fibrosis-preventing efficacy of which had been substantiated in an earlier clinical study [19]. In this subgroup of eyes, the impact and potential advantages of this additional treatment was evaluated. Polishing was performed after completion of cortex aspiration and meticulous peeling of any residual lens fibers adhering to the peripheral posterior capsule.

\section{Results}

Surgical complications In a preliminary evaluation of the first 376 consecutive cases performed during the first year (Menapace R. Routine posterior optic buttonholing for after-cataract prevention: The better alternative to the sharp

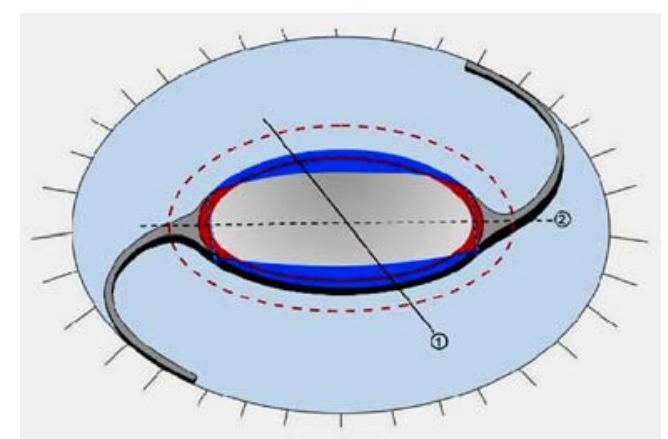

Fig. 2 Schematic of interrelationship between capsule leaves and IOL. Loops residing in capsular fornix, optic buttoned-in in PPCCC. Posterior capsule lying on top of optic (crescent-shaped blue areas); this precludes optic contact and thus fibrosis of the anterior capsule (red lines: rim of ACCC). If the ACCC is smaller than the optic (bold line), anterior capsule fibrosis limited to area adjacent to the haptic junction (red area); if larger than the optic (hatched line), no fibrosis also in this area 
Fig. 3 Retroillumination photography of buttoned-in IOLs (HOYA AF-1): note perfect autocentration of optic and run of posterior capsule rim
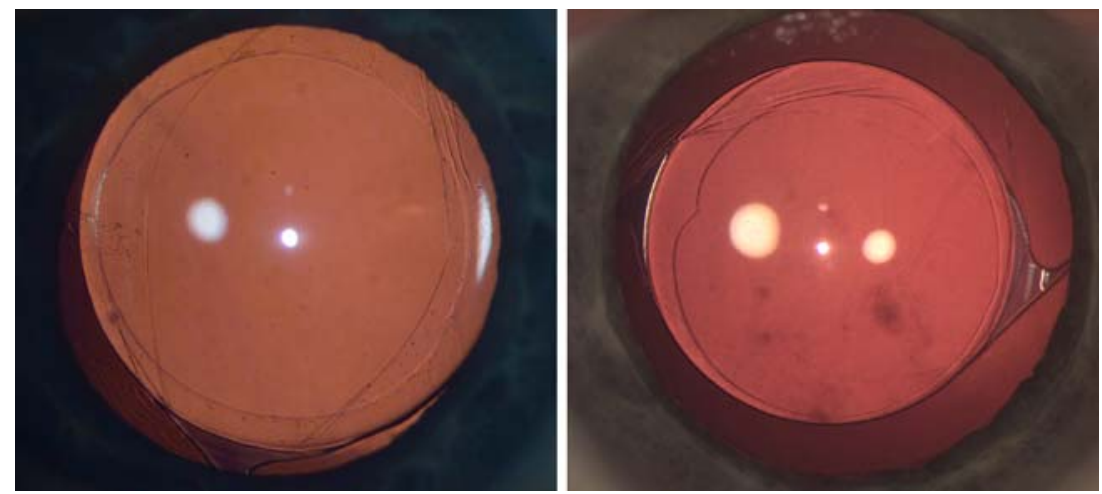

edge optic? Breaking News Session, XXIIIth Congress of the ESCRS, 10.-14.9.2005, Lisbon) no case of cystoid macular edema (CME), and only one case of retinal detachment (RD) were encountered. Since the latter occurred only 4 months after surgery in a highly myopic eye with an intact anterior hyaloid surface, the causative role of $\mathrm{POBH}$ is questionable. The $\mathrm{RD}$ emerged from a group of small round holes in the superotemporal quadrant, and was repaired by cryopexy and gas injection. In only three cases could buttoning-in not be performed as planned because of a too small or decentered PPCCC. However, even with a suboptimally centered PPCCC the optic always perfectly centered permanently. During surgery, there was no case of vitreous prolapse into the anterior chamber, and therefore no need for an anterior vitrectomy. Postoperatively, four eyes presented with a minor, and two eyes with a major vitreous entrapment without apparent vitreous traction. These cases happened at a time when the surgical procedure was still being elaborated, and the importance of performing a complete circumferential viscodissection of anterior hyaloid and posterior capsule prior to the optic buttoning-in manoeuvre to preclude vitreous incarceration was not yet fully recognized. One of the two patients with major entrapment underwent bimanual anterior vitrectomy in topical anesthesia through the pre-existing paracentesis openings while the optic was temporarily desenclavated. The other patient declined the proposed reintervention. No CME or RD ensued in any of these eyes in the 3-year follow-up period. The technique, results, and complications of the first 500 consecutive cases have been extensively published in a peer-reviewed journal [12]. In a later subseries which investigated the effect of creating an ACCC
Fig. 4 HOYA AF-1-type IOL preferred because of continuous haptic-optic junction and "hopper loop" haptic. POBH causes optic to position (by approximately $1 \mathrm{~mm}$ ) more posteriorly (compared to standard in-thebag placement), thereby stabilizing the vitreous body
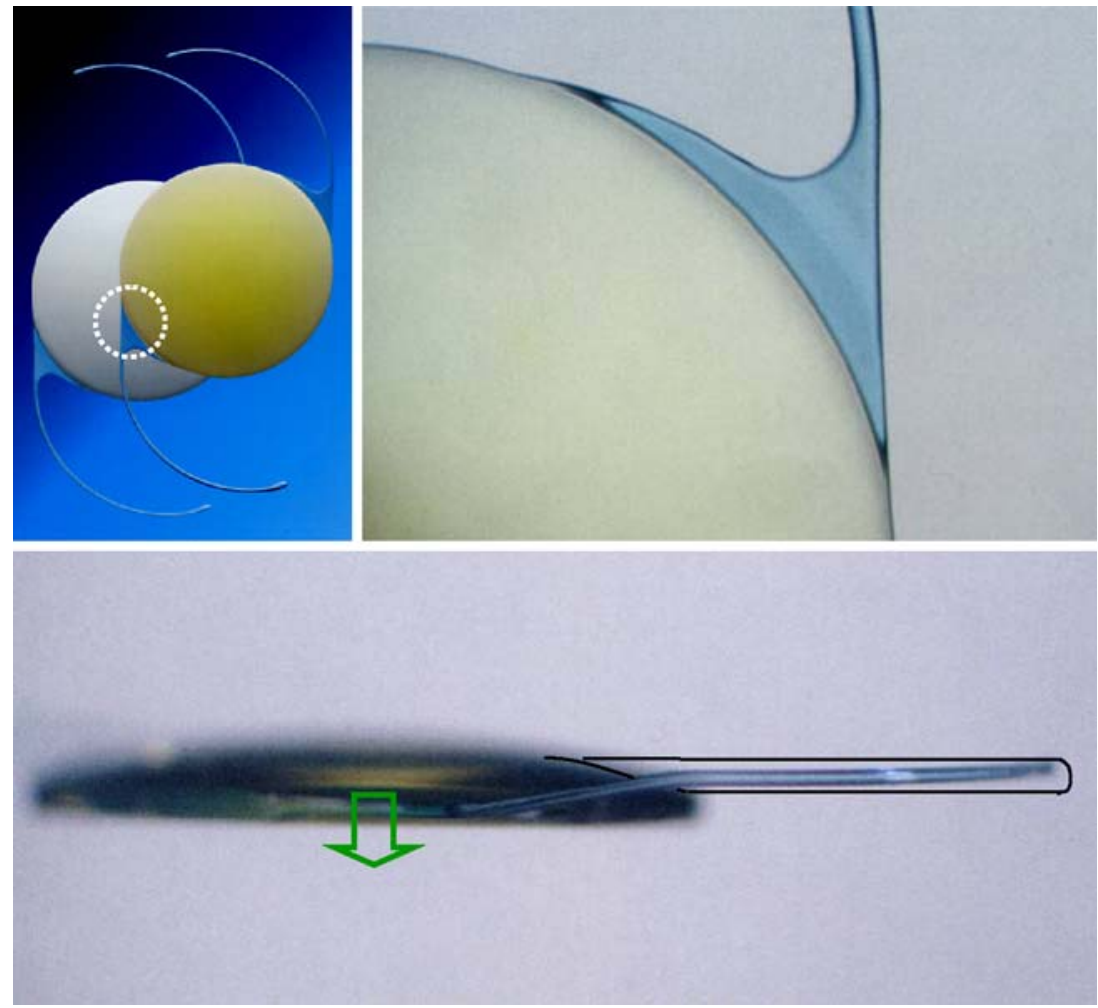
Fig. 5 "Aspiration curette" for effective and safe debridement of anterior LEC layer additionally reduces capsular fibrosis
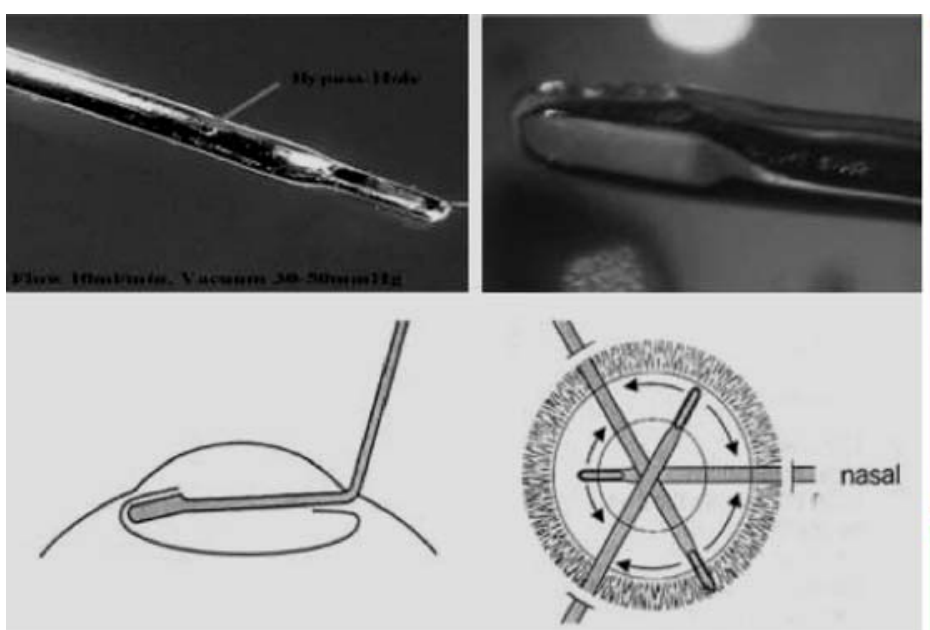

Dependency of actual vacuum level on flow rate (vacuum limit $50 \mathrm{mmHg}$ )
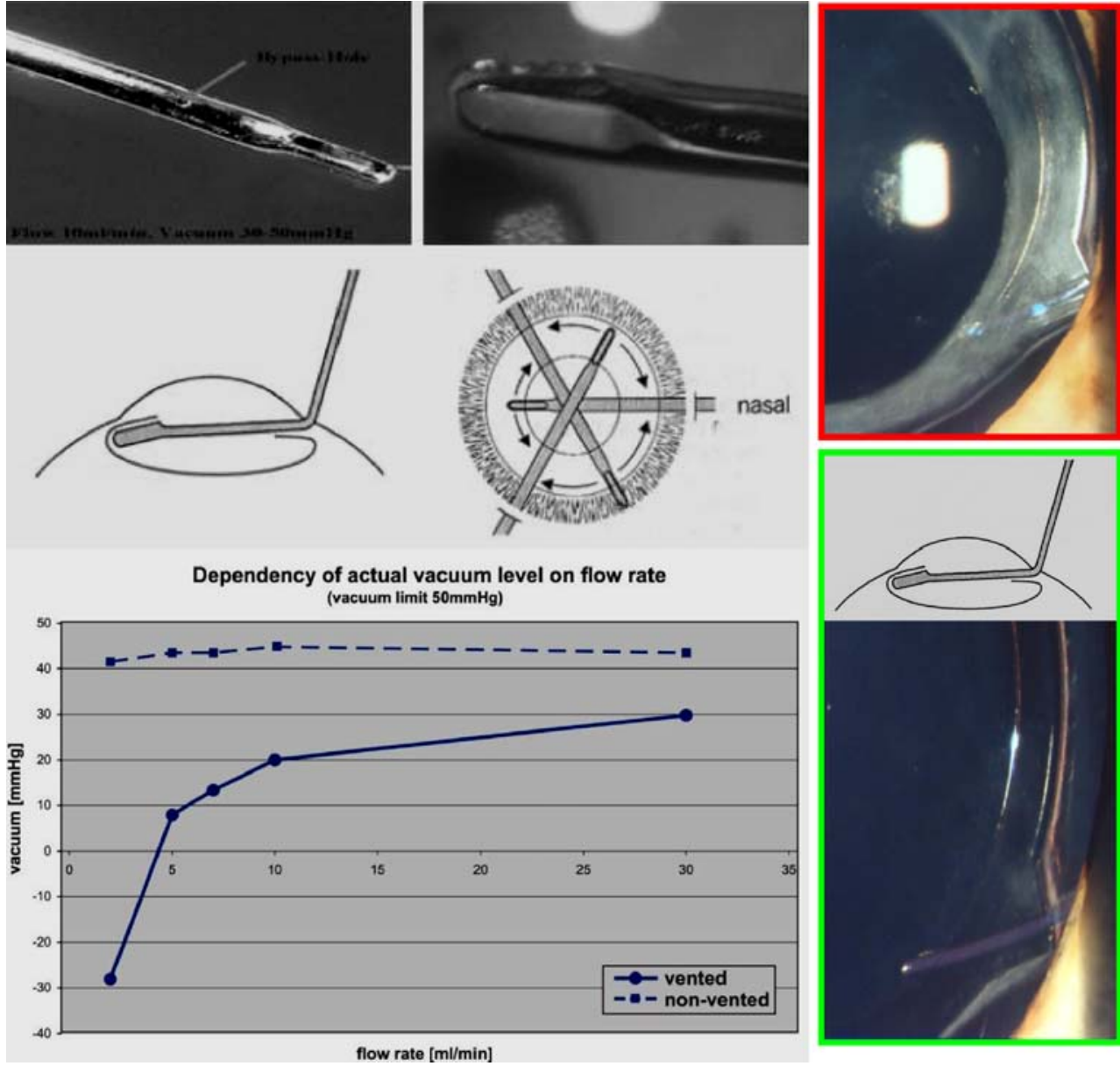

opening larger than the optic diameter to further reduce anterior capsule fibrosis especially at the haptic-optic junction, two other cases of delayed vitreous herniation into the anterior chamber occurred when a relatively large but still overlapping PPCCC was secondarily distended by posterior capsule contraction due to fibrosis emerging from the edge of the ACCC rim outside the optic, thus exposing the optic edge and creating a gap in the capsular diaphragm along the optic rim (Fig. 6). Consequently, due to the saccadeous eye movements, mobile vitreous incrementally crept into the anterior chamber. Using bimanual vitrectomy, with the infusion inserted through one of the pre-existing paracentesis openings and the vitrectome through a pars plana sclerotomy, the floppy vitreous strand was retracted into the vitreous cavity and excised. Except for these few cases, no further cases of vitreous entrapment or herniation were encountered in the second 500 consecutive cases. In two other cases of the above-mentioned sub-series, spontaneous desenclavation of the optic without vitreous presentation was noted the day after surgery due to a too largely dimensioned PPCCC. There was no case of endophthalmitis in the 1000 cases performed up to June 2007. The results of the first 150 cases - which may represent the learning curve — and the subsequent 850 cases are summarized in Table 1.

After-cataract preventive effect After a maximum followup exceeding 3 years, there was no case of retrolental aftercataract formation. The optic inside the capsular edges

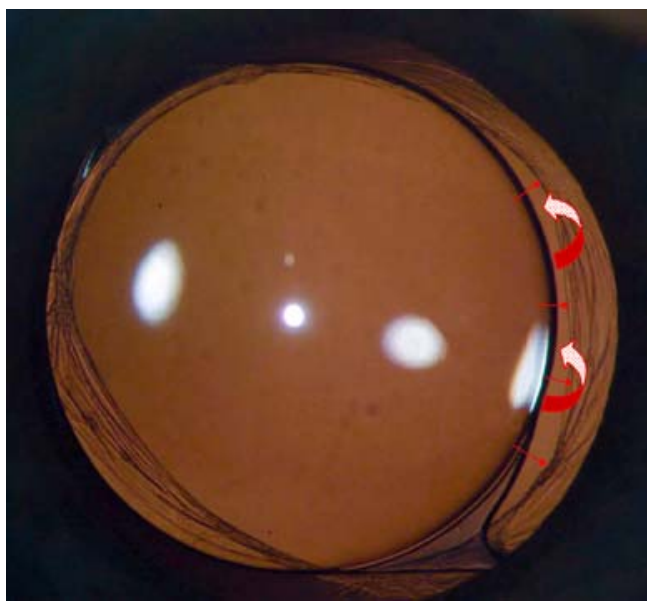

Fig. 6 Retraction of scarcely overlapping PPCCC rim from optic rim due to fibrosis emerging from ACCC larger than optic allows for delayed vitreous herniation 
Table 1 Complications

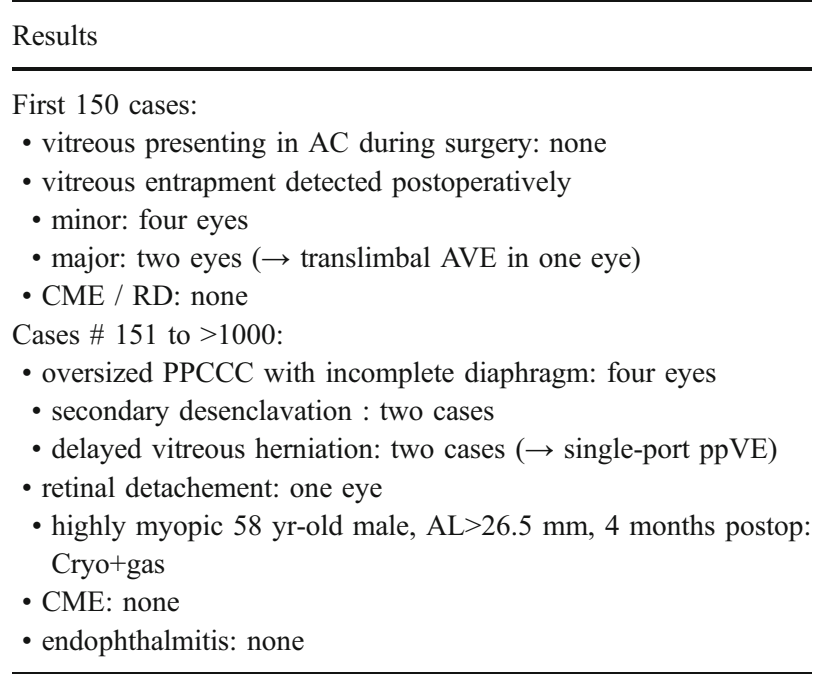

remained completely clear in all cases (Figs. 7 right and 8). When the anterior capsule was left unpolished, the posterior capsule overlying the optic periphery was often covered with a thin layer of translucent regeneratory LECs that formed into delicate pearls. These, however, did not relevantly compromise visualization of the peripheral retina. In some cases, localized Soemmering's ring formation was observed (Fig. 8 left). Additional anterior capsule polishing significantly reduced regeneratory LEC proliferation on the residual posterior capsule, thereby optimizing visibility of the peripheral retina (Fig. 8 right). Fibrotic after-cataract formation depended upon whether anterior polishing had been performed or not. If so, no fibrosis was observed at all. If not, fibrosis of the anterior capsule was essentially restricted to the area adjacent to the haptic junction, where the undercrossing posterior capsule allowed an anterior capsule smaller than the optic diameter to establish direct contact with the optic (Fig. 9 and 10). From there, limited encroachment of the fibrosis unto the neighboring capsular areas was often noted. Typically, some amount of fibrosis was observed to have spread out along both the anterior and posterior capsulorhexis edges. In the areas between the haptic junctions where the posterior capsule overlapped the optic and had been sandwiched between the latter and the anterior capsule, however, both capsules remained essentially clear and transparent. If the anterior rhexis was larger than the optic, no fibrosis formed, also adjacent to the haptic junctions, even without capsular polishing due to the lack of contact to the optic. However, fibrosis extending from the ACCC edge unto the posterior capsule retracted the latter from the optic edge in two cases with scarce optic overlap, resulting in gaping of the capsular diaphragm and delayed vitreous herniation as reported above.

Detailed risk profile Several substudies were initiated to exactly determine potential risks compared to the standard in-the-bag implantation technique. All were prospective randomized intraindividual comparison studies. There was no statistically significant difference in the early postoperative pressure course, either with or without the use of pressure-lowering drops [21]. This was also true for the inflammatory response as measured with a laser-flare-cellmeter, even after inadvertent puncturing of the anterior hyaloid surface [20]. Regarding the postoperative axial shift of the IOL optic and its final position, there was a significant difference. While during the first 4 weeks the bag-fixated optics generally moved forward to finally settle more anteriorly, the buttoned-in optics immediately stabilized and did not move thereafter. This resulted in a difference of mean anterior chamber depth of approximately $1 \mathrm{~mm}$. Accordingly, there was a significant mean myopic shift after bag-fixation, while refraction remained unchanged after buttoning-in of the optic [22]. Though no clinically apparent CME was reported, subclinical postoperative changes had to be ruled out. This was done by investigating the thickness and morphology of the central retina using a last-generation optical coherence tomography (OCT) device. In a series of 50 bilateral cases, no difference could be found in any of these parameters (Menapace R, Stifter E. Macular integrity after cataract surgery with combined primary posterior capsulorhexis and posterior optic buttonholing as evaluated by optical coherence tomography. Abstract XXVth Congress of the ESCRS, 8. -12.9.2007, Stockholm), [23]. Another ongoing study is
Fig. 7 Right. Two years after POBH: optic within capsule edges completely clear, posterior capsule overlying optic shows thin layer of regeneratory LEC proliferation with scarce small pearls. Left. Contralateral eye with in-the-bag placed IOL: regeneratory LEC proliferation on posterior capsule behind optic with abundant large pearls
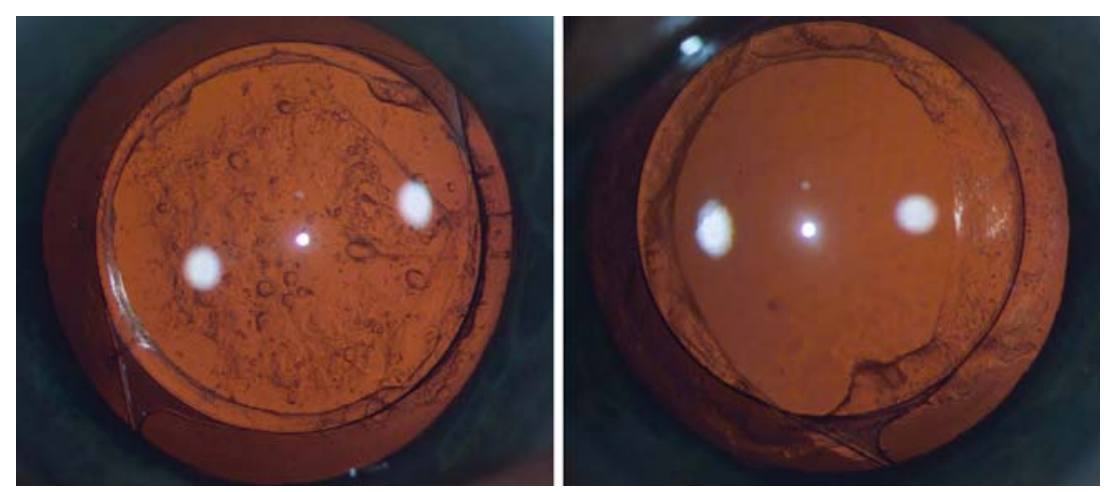
Fig. 8 Right. Two years after POBH with polishing: significantly lower amount of regeneratory LEC proliferation-compared to contralateral eye (left) having undergone POBH without polishing
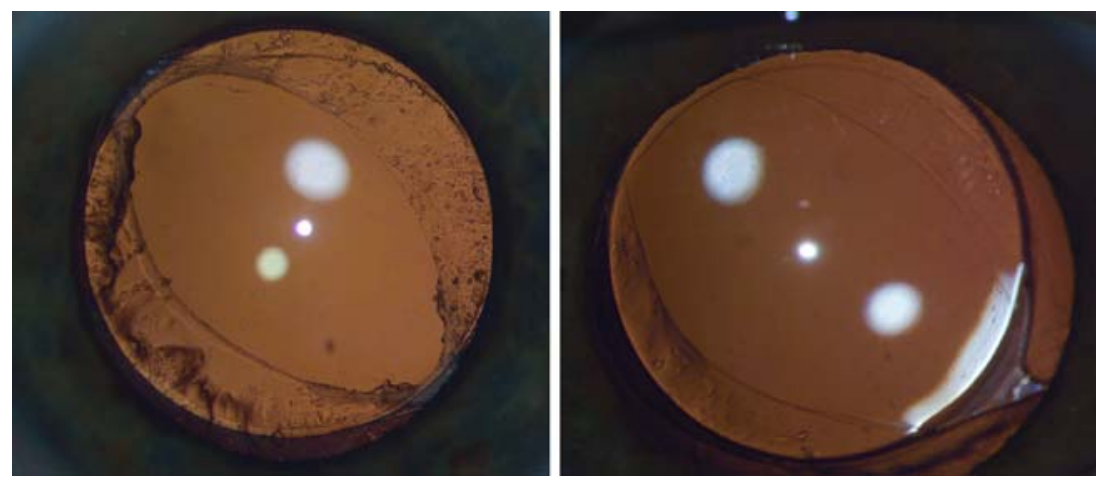

proving the clinically obvious lack of optic decentration and tilt even within a suboptimally centered PPCCC.

\section{Discussion}

The advantages, possible downsides, and specific features of the technique are addressed in the following:

The advantages of PPCCC fixation of the optic as compared to the standard in-the-bag fixation were found to be:

1. Eradication of after-cataract formation behind the optic by diverting migrating LECs to bypass the optic rim towards the anterior optic surface. When the optic is buttoned-in, the posterior capsule leaf is relocated to lie on top of the anterior optic surface (Figs. 2, 3 and 8). By principle, migrating LECs can no longer access the retrolental space. At the optic junctions where the posterior capsule leaf undercrosses the haptic base, the high pressure exerted by the distended posterior capsule ring blocks LEC migration beneath the junctions.

2. Significant reduction of capsular fibrosis without polishing. Myofibroblastic transdifferentiation is the substrate of capsular whitening and shrinkage. It

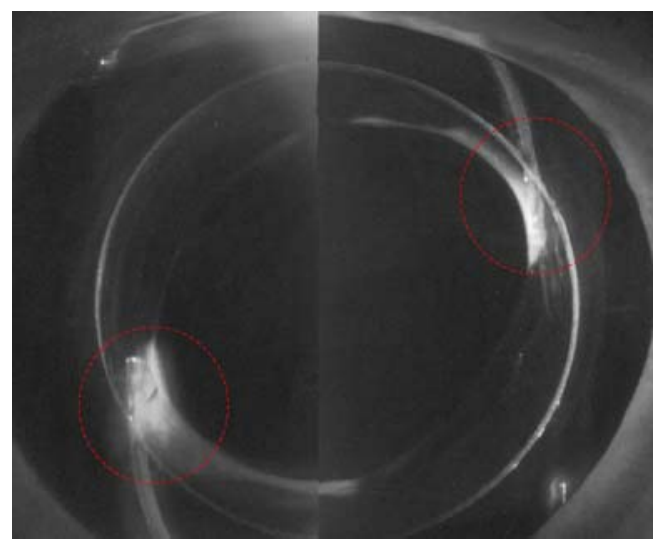

Fig. 9 Posterior capsule sandwiched between anterior capsule and optic precludes direct contact which catalyses anterior LEC transdifferentiation leading to fibrosis. Residual fibrosis essentially confined to haptic-optic junction mainly affects the anterior capsule, and is the cause for numerous complications including rhexis contraction ("rhexis phimosis") and rhexis retraction with secondary extrusion of the optic out of the bag ("anterior optic buttonholing"), with possible decentration and tilt [10]. Direct contact between the anterior capsule and the optic is the trigger mechanism. The optic material then catalyses the myofibroblastic transdifferentiation of the anterior LECs on the back side of the anterior capsule, with consecutive capsule contraction and collagen deposition [8]. Buttoning-in causes the posterior capsule to be sandwiched between the anterior capsule and the optic, avoiding direct contact between the two. Consequently, LEC transdifferentiation of the anterior LEC layer is not initiated ("no contact - no fibrosis"). Only along the capsulorhexis edges some residual fibrosis may be seen. The usually seen extensive fibrosis of the whole anterior LEC layer remains restricted to the area of direct optic contact inside the optic junction.

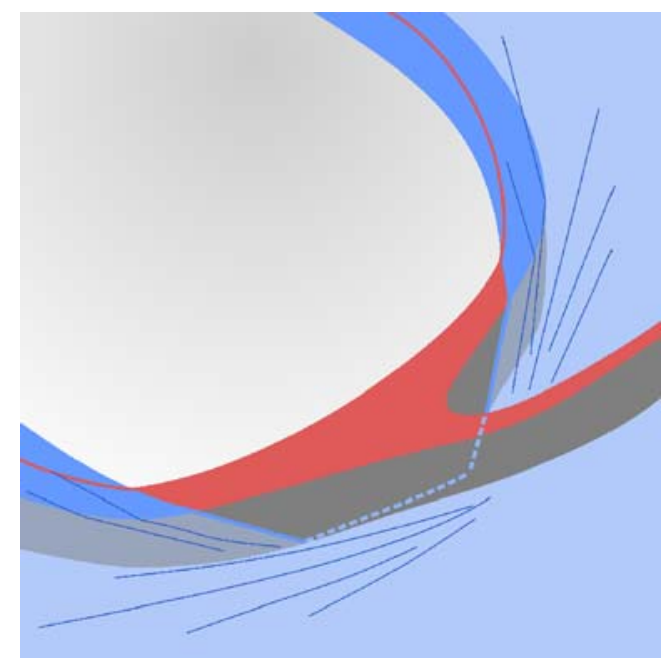

Fig. 10 Schematic close-up view highlighting capsule-IOL interplay at haptic junction. Undercrossing of PPCCC edge allows for localized anterior capsule contact with optic, resulting in fibrosis adjacent to haptic junction which may spread out along rhexis rim 
3. Reduced anterior optic ongrowth without polishing. After standard in-the-bag fixation, ongrowth of LECs unto the free optic zone is regularly observed with acrylates to a variable extent [1]. Usually transient, it may also be permanent. Though mostly limited to the periphery, it may in cases cover the whole free optic zone. With POBH this complication is abolished, since the crescent-shaped projection of posterior capsule forms a buffer zone between the anterior rhexis edge and the free optic. As with fibrosis, the optic ongrowth is restricted to the areas of direct anterior capsule contact to optic inside the haptic-optic junction.

4. Eradication of residual fibrosis and ongrowth with additional polishing. Additional polishing of the anterior capsule specifically avoids fibrosis or ongrowth inside the haptic-optic junction, and fully excludes residual fibrosis in the areas where both capsules overlap.

5. Capsular polishing without negative impact of retrolental regenerate formation. With bag-fixated IOLs, the strength and permanence of the barrier effect at the optic edge is largely dependent upon fibrosis [10]. Polishing, though effectively reducing fibrosis, therefore results in a significant increase of the YAG-LCT rate due to weakening collagenous edge sealing [11]. Since with POBH the migrating LECs are redirected to the anterior optic surface instead of being halted at the optic rim, the after-cataract preventive effect is completely independent of fibrosis. Polishing, therefore, does not promote retrolental LEC ingrowth when combined with POBH.

6. Autocentration of optic. Even with a suboptimally centered PPCCC, the optic will automatically center within the buttonhole and align with the anatomical axis of the eye (Fig. 11), since the centering force is exerted by the loops which reside in the capsular bag fornix. As opposed to standard capsular bag fixation, centration of the optic is also independent of the size and centration of the ACCC.
7. Forgivingness of undersized PPCCC. Due to the extreme stretchability and elasticity of the posterior capsule, $\mathrm{POBH}$ will still be feasible with a PPCCC opening smaller than $4 \mathrm{~mm}$ in diameter (Fig. 12). If the PPCC opening should happen to be too small to allow for buttonholing, the PPCCC can be either secondarily enlarged (similar to an ACCC), or the optic is simply placed in the bag without being buttoned in. In fact, this only happened in the early series in cases with a poorly dilating pupil.

8. Immediate stability of capsular diaphragm and implant. Due to the lack of forward movement of the optic as seen after in-the bag fixation due to the loss of haptic memory and the distension of the capsule diaphragm as the capsules seal and fibrose, no myopic refractive shift occurs. While delayed by up to 4 weeks after bagfixation depending upon the haptic design and material, final refraction is thus instantly attained, allowing prescription of final glasses 1 week after surgery, when the patient is seen by the referring ophthalmologist.

9. Posterior positioning of the IOL optic by about $1 \mathrm{~mm}$ compared to bag-fixation significantly increases the interspace between the iris and optic. This avoids chronic iris chafing when a sulcus-fixated add-on IOL is secondarily implanted in order to correct for an erroneous IOL power selection, or to add astigmatic correction or multifocality.

Two advantages of POBH deserve special mentioning:

1. After-cataract prevention is entirely independent of the optic edge design. Thus, the design of the optic rim can be optimized in order to minimize reflectivity and thus dysphotopsia [16] without compromising the after-cataract performance of the IOL.

2. After-cataract prevention is entirely independent of the optic material. Thus, the choice of optic material can be solely driven by achieving optimum biocompatibility, leading to minimum cell and bacteria
Fig. 11 Bag-fixated haptics compensate for decentration of PPCCC opening
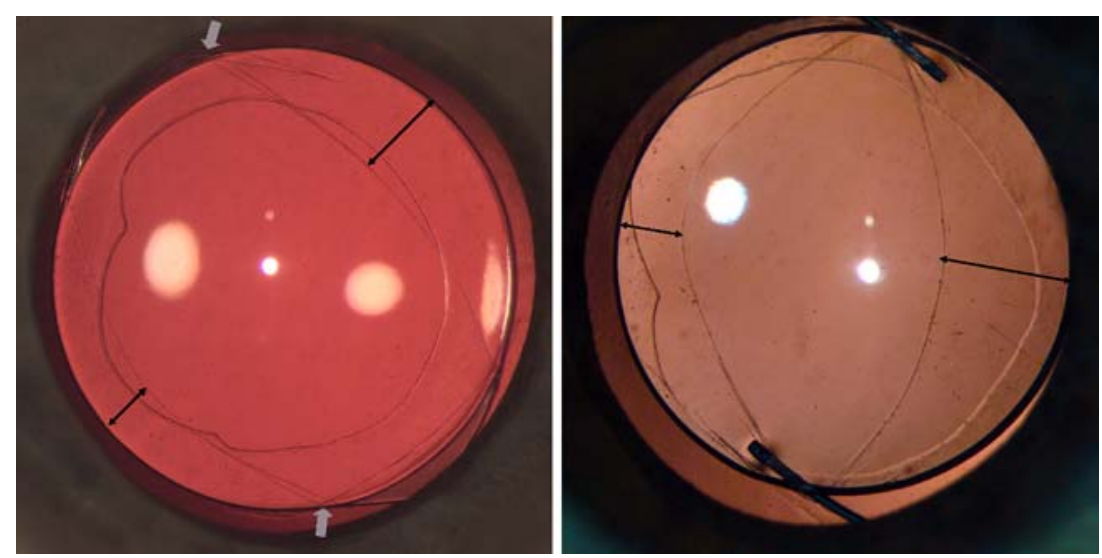
Fig. 12 Exquisite stetchability and elasticity of posterior capsule compensates for undersizing of PPCCC opening
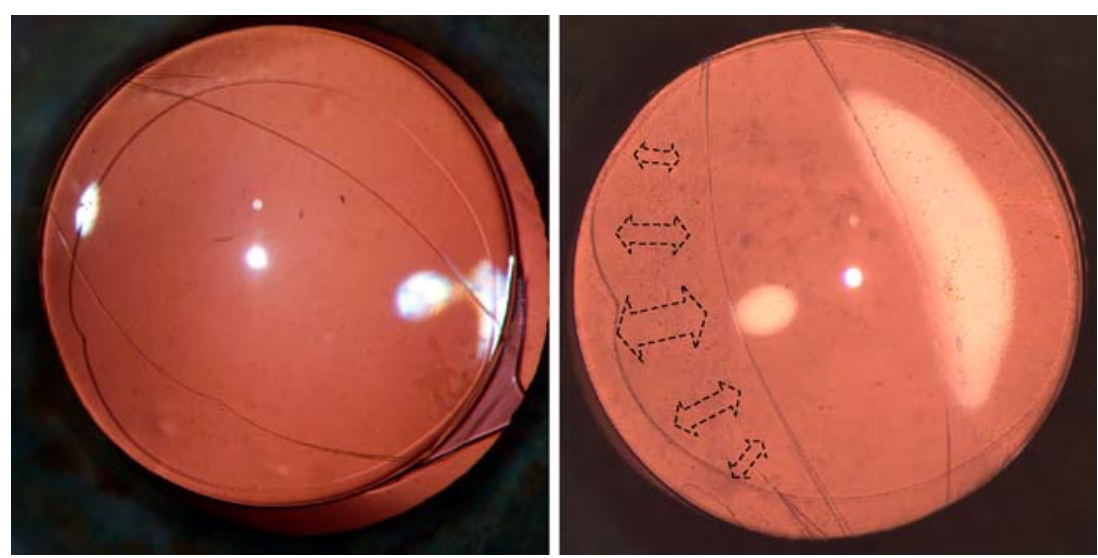

adhesion. This may bring about a renaissance of the round-edged optics. Also, efforts in improving IOL materials may solely concentrate on optical performance, biostability and biocompatibility irrespective of their LEC inhibiting properties.

As opposed to PPCCC with bag-fixation of the optic, $\mathrm{POBH}$ creates a stable and watertight diaphragm. Vitreous prolapse or entanglement will not occur should the anterior chamber flatten after retracting the aspiration-infusion tip following removal of residual OVD from the anterior chamber.

The POBH technique as such is safe and highly effective with regard to after-cataract prevention. It is not only feasible, but highly recommended to perform it under topical anesthesia in order to guarantee bright and stable retroillumination when performing the PPCCC. No special or extra instruments or implants are required.

Interestingly, no case of endophthalmitis occurred in the whole series, despite the fact that no intracameral or postoperative antibiotic prophylaxis had been performed. Though this series is not large enough to draw a meaningful conclusion, it may be attributed to the fact that buttonholing avoids seclusion of bacteria within the retrolental space. Other than with bag-fixation, the optic and capsule surface are fully exposed to the aqueous humor circulation from both sides. In case of an endophthalmitis, the vitreous cavity may be easily accessed for translimbal tapping and bimanual vitrectomy after temporary desenclavation of the IOL optic.

Four crucial surgical steps deserve in-depth discussion:

1. Sizing of the ACCC. Apart from capsular polishing, formation of residual fibrosis, in particular adjacent to the haptic-optic junction, can also be avoided by making the ACCC larger than the optic diameter (Fig. 13 right). However, combining a standard $5 \mathrm{~mm}$ ACCC with anterior capsule polishing is definitely recommended over creating an overly large ACCC, because of the following downsides of the latter. Appropriate dimensioning of the PPCCC is rendered more difficult since the ACCC can no longer be utilized as a ruler, making unintentional under- or oversizing more likely. Should a PPCCC result to be much larger than the optic, alternative buttoning-in of the IOL optic through the ACCC opening can then no longer be resorted to. Also, a posterior capsule that only slightly overlaps the optic may be pulled off its edge as fibrosis of the anterior capsule sets in. Delayed gaping of the capsule-IOL diaphragm along the optic rim, with possible consecutive vitreous herniation, may result, as has happened in two cases. Therefore, a 5-mm ACCC combined with anterior capsule polishing is definitely preferable over creating an oversized ACCC.

2. Viscodissection of the central hyaloid-capsular interspace (Berger's space and Wieger's ligament).

In the young eye, the anterior hyaloid surface is separated from the posterior capsule by an interspace with two concentric compartments: Berger's space in the center, and Girard's space in the periphery. These two compartments are separated by a concentric circular ring along which both membranes are attached to each other, the Wieger's ligament, which has a diameter of 8-9 $\mathrm{mm}$ and a width of 1-2 mm (Fig. 14). In practice, a great anatomical variability is experienced while performing a PPCCC in adult or senile eyes. In some eyes, attachment along Wieger's ligament is no longer present, with the two united compartments thus forming one commun patent interspace. In others, there is hardly any interspace present, and viscoseparation of the strongly adhering membranes may be difficult to perform. This may be very difficult or not at all anticipated using the slitlamp with a cataractous lens. Therefore, utmost care must be used when centrally incising the posterior capsule, and inadvertent puncturing of the anterior hyaloid membrane still cannot be fully excluded. If in such a case larger quantities of OVD were injected through the capsular opening immediately after puncturing, the OVD might find its way through the punctured hyaloid surface and spread behind it within the vitreous body. This is excluded by performing part of the PPCCC beforehand. Only then, OVD 
Fig. 13 Schematic detailing capsular interplay with ACCC smaller (left) and larger than optic (right). Creating an ACCC larger than the optic precludes contact and thus fibrosis at the haptic-optic junction (green circle), but may retract a scarcely overlapping PCCC and thus expose optic rim

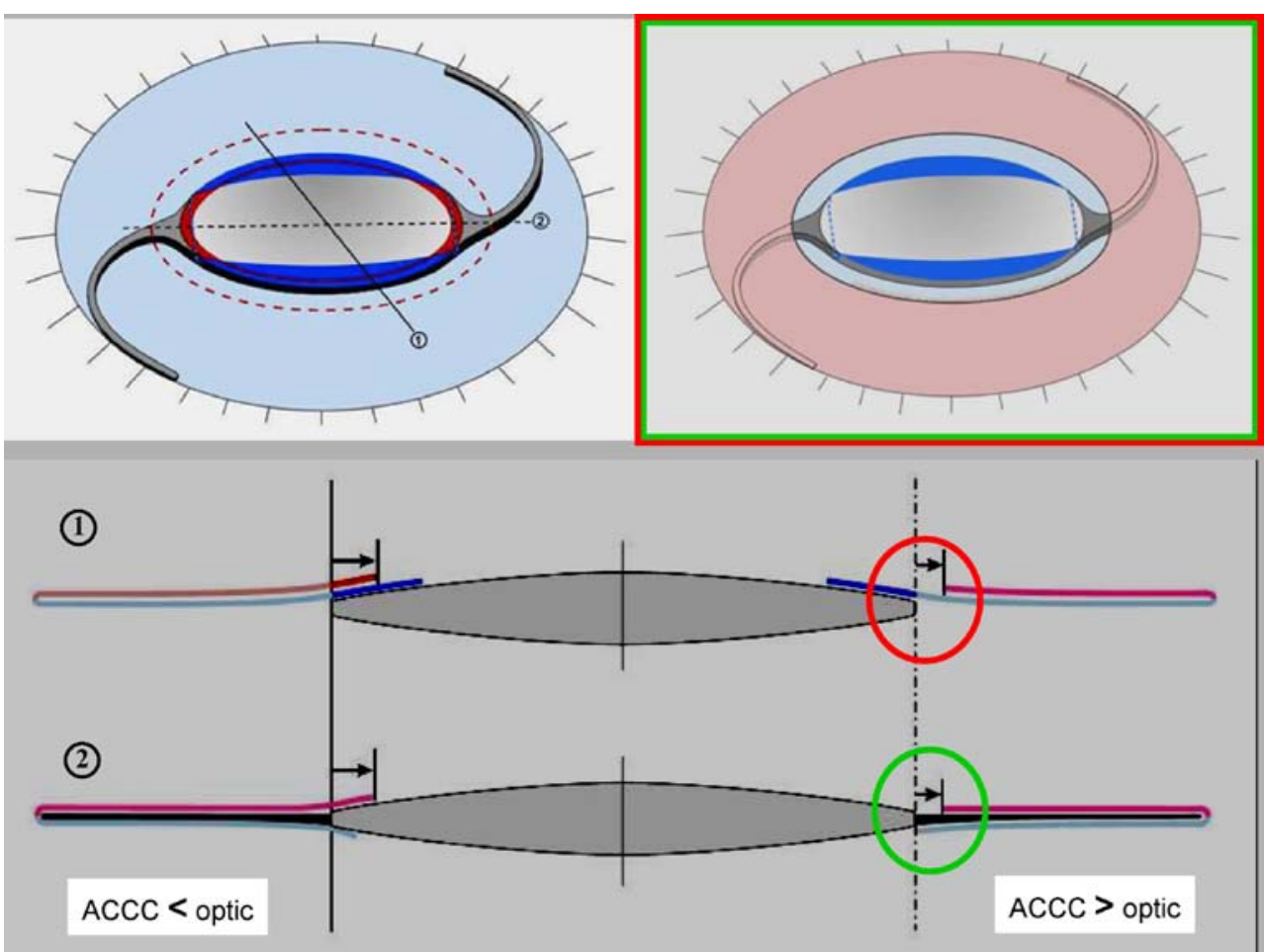

is injected into Berger's space to ensure that separation of the anterior hyaloid surface from the capsule has been attained. Viscodissection is then continued until Wieger's ligament, if still adherent to the capsule, is severed and Girard's space peripheral to it is accessed.

\section{Sizing and centration of the PPCCC.}

The posterior capsule being extremely stretchable and elastic, buttoning-in will also be feasible when a PPCCC turns out to be smaller than $4 \mathrm{~mm}$. Also, the forces of the haptics will center the optic unto the anatomical axis within a suboptimally centered PPCCC. Therefore, though a $5 \mathrm{~mm}$

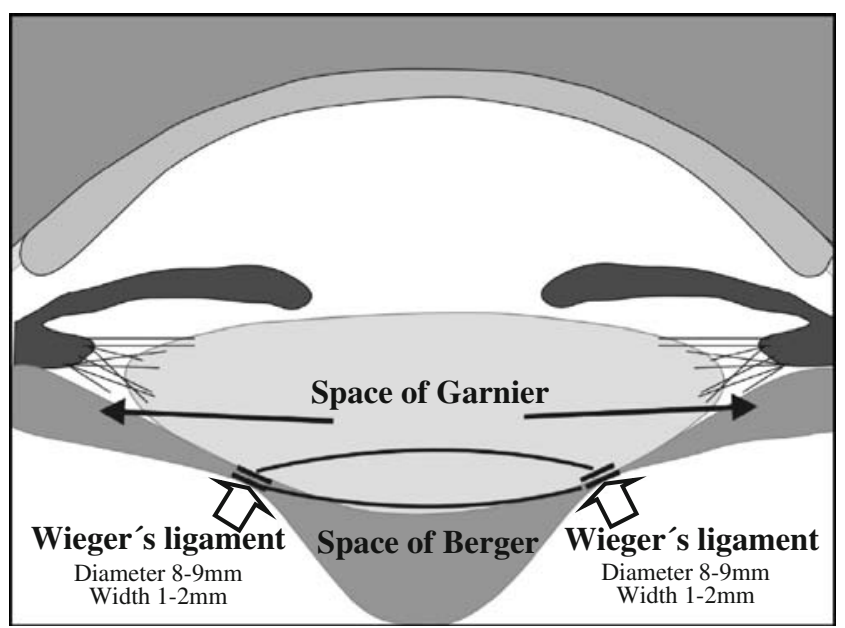

Fig. 14 Schematic depicting interrelationship between posterior capsule and anterior hyaloid (Courtesy of Dr. T. Miyoshi). In practice, Wieger's ligament may be completely detached; conversely, hyaloid may be closely adhering to capsule over extended areas diameter and perfect centration should always be aimed at, deviation from this target will be tolerated. However, with a too large PPCCC that evades the optic rim, even when only along part of optic circumference, the IOL must be buttonholed through the ACCC with the loops either placed in the sulcus or capsular bag equator in order to avoid delayed vitreous prolapse. Therefore, the ACCC should be well-centered and no larger than $5-6 \mathrm{~mm}$ in diameter, to preserve the option of anterior rhexis fixation of the optic. Apart from providing an alternative for safe IOL fixation, an appropriately dimensioned ACCC also serves as a ruler for adequate sizing of the PPCCC.

Sizing of the PPCCC may be difficult in eyes with a large capsular bag or lax zonules. This is heralded by wrinkles in the posterior capsule when flattened by OVD, and by traction folds when trying to create a central puncture or when pulling on the capsule edge when starting out with the PPCC. In such a case, implantation of a capsular tension ring remedies the problem as it puts the posterior capsule on radial stretch, creating uniform vector forces along the whole circumference. Such a ring can still be safely implanted when the PPCCC has already been started. However, bimanual tangential insertion should be preferred over injector implantation, and a smaller ring with a low resilience should be chosen. This minimizes capsular stress and distortion of the anterior segment [9], which could potentially lead to extension of the PPCCC or protrusion of the vitreous surface.

4. Viscodissection of the peripheral hyaloid-capsular interspace (Girard's space). 
Before implanting the IOL, the peripheral interspace between posterior capsule and anterior hyaloid must be circumferentially dissected with OVD in preparation of optic buttonholing. If not appropriately performed, the hyaloid surface may be shoveled up and damaged by the rim of the optic.

5. Selection of IOL design. Though 1-piece IOLs with broad loops have been successfully used for the procedure [12], 3-piece IOLs with slim loops are definitely preferable. Of these, the HOYA AF-1 is most appropriate, since its continuous transition between haptic and optic allows the rim of the PPCCC to smoothly slide along it while the IOL is buttoned-in and centers itself within the PPCCC opening (Fig. 15)

Are there any drawbacks of the PCCC plus POBH concept long-term with regard to delayed after-cataract formation or retinal complications?

1. Delayed after-cataract formation. In principle, equatorial LECs might access the retrolental space beneath the haptic junction where the PCCC rim under-crosses the haptic base. This has been observed in some cases, but was always minimal and limited to the very periphery. The explanation may be that the stretched PPCCC rim firmly adheres to the haptic base, and LEC migration is very much limited, due to the narrow door for potential

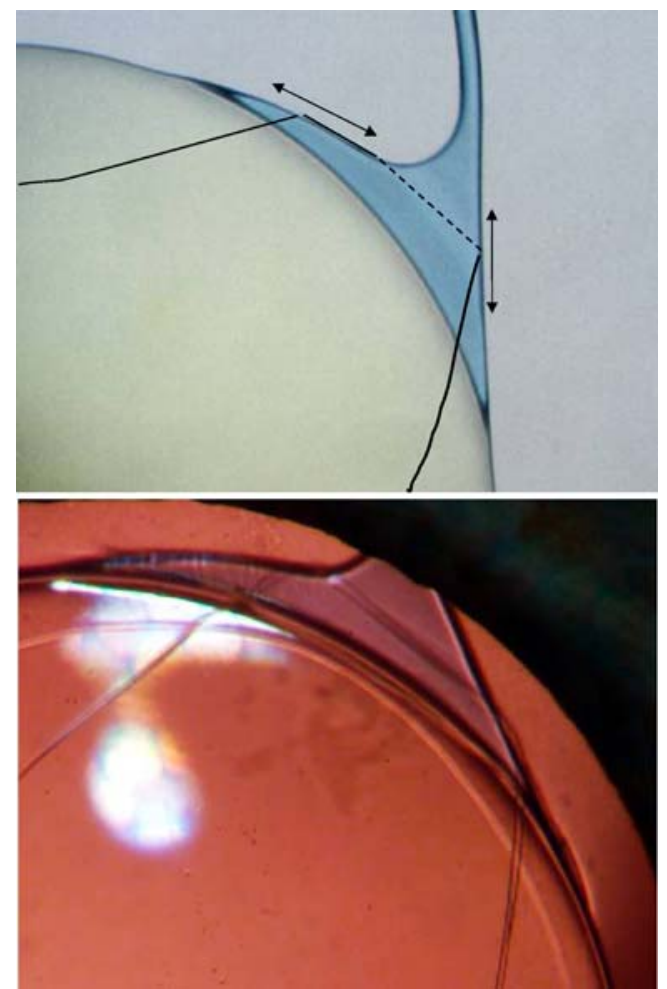

Fig. 15 Continuous haptic-optic transitions allow PPCCC rim to smoothly slide along IOL circumference as optic is buttoned-in and centered within PPCCC by loops residing in capsular bag fornix invasion and the lack of capsular as a scaffold for migration should the barrier fail. Moreover, because of the lack of collagenous sealing between the capsular leaves LECs might theoretically invade the exposed posterior capsule and form pearls that accumulate at the anterior capsular rim or protrude into the anterior chamber, as is seen along the edges of YAG-LCT openings. This, however, has also not been observed to a clinically significant extent. LEC ongrowth unto the exposed posterior capsule, if at all present, was always flat with no or only hinted pearl formation not exceeding the PPCCC rim. Both observations have been recently confirmed by a pertinent publication on the eyes of children who had undergone a similar procedure 5 to 12 years earlier [7], which may be explained by the proliferation-inhibiting effect of the aqueous. Considering the fact that children's eyes exhibit a much higher proliferative potential, long-term results in adults may be even better.

2. Retinal complications. An increased incidence of CME and $\mathrm{RD}$ rate has been a concern. An earlier report did not find an increased incidence with PPCCC alone [26]. In our series of over 1000 POBH cases performed so far, not a single case of clinically significant CME was reported, and our OCT study revealed no difference in macular thickness or morphology.

CME is provoked by the dissipation of cytokines released in the anterior segment, and by vitreous traction exerted by the anterior displacement of the vitreous body following exchange of the natural lens volume by a thin artificial lens. In these regards, POBH has certain advantages over standard in-the-bag IOL implantation. Firstly, as opposed to a sole PPCCC, optic buttoning-in creates a watertight diaphragm blocking the posterior dissipation of cytokines. In addition, viscodissection of the posterior capsule and anterior hyaloid creates a contiguous cushion of OVD behind the capsule-IOL diaphragm including the zonular region which precludes access of cytokines to the posterior segment until it is eventually resorbed. The more posterior positioning of the buttonholed optic together with the OVD cushion behind it, and the lack of subsequent anterior movement of the optic, prevent both immediate and delayed anterior displacement of the vitreous body. Severing the attachments between the posterior capsule and the hyaloid, namely Wieger's ligament, may help to avoid traction on the vitreous when the chamber happens to flatten during OVD aspiration, or thereafter.

Regarding RD, one case was encountered 4 months after surgery in a young male with high axial myopia. For standard cataract and IOL surgery, a recent study reported a 5 -year RD incidence of $0.7 \%$ and $21 \%$, depending on the absence or presence of lattice degenerations [18]. In the 


\section{Toric IOL}

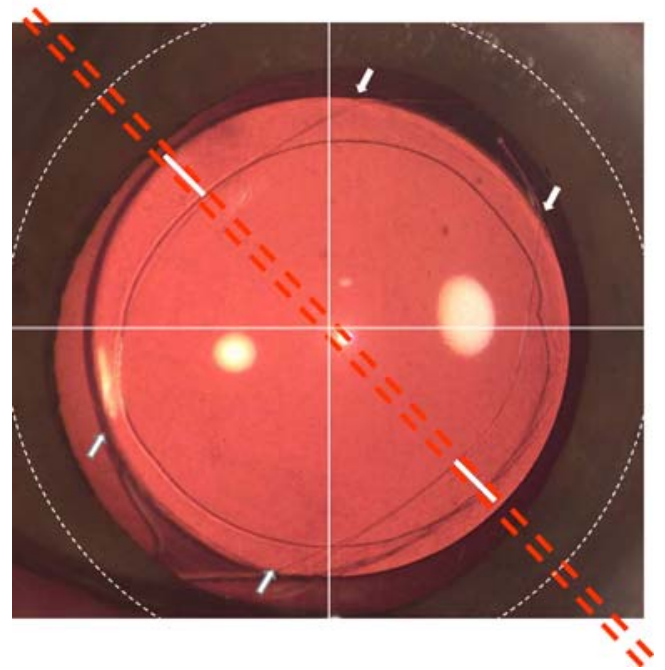

Fig. 16 Rhexis-fixation precludes postoperative IOL rotation in an oversized bag and delayed optic rotation by haptic compression arising from capsular bag shrinkage

5 years following standard cataract surgery, such posterior vitreous detachment occurs in about four out of five eyes with the vitreous still attached. This is triggered by the anterior positioning of a bag-placed optic. Though our follow-up with POBH surgery is shorter than 5 years, the excessively low RD rate of only $0.1 \%$ overall seems to indicate a significantly lower risk. This may explained as follows: as an IOL optic buttoned-in into a PCCC is positioned $1 \mathrm{~mm}$ more posteriorly compared to bag-fixation [22] (Fig. 4 bottom), it inherently stabilizes the vitreous, and thus may avoid or delay posterior vitreous detachment.
Table 2 Indications

Summary of indications

[Pediatric cataracts]

PEX-Syndroma

High myopia (reduced barrier effect)

Peripheral retinal disease: axial myopia, diabetes (improved insight for diagnosis \& therapy)

Multifocal IOLs (patients more susceptible to capsular opacification) $\rightarrow$ Toric IOLs

$\rightarrow$ Accommodative lens system

Severing of all adhesions between the posterior capsule and the anterior hyaloid and creating a cushion of OVD behind the capsule-IOL diaphragm, which is only slowly resorbed, may serve as an additional protective factor. A longterm study, including the incidence of vitreous and retinal detachment following $\mathrm{POBH}$, is required to establish possible advantages of this technique over standard in-thebag implantation of IOLs.

If all the precautions detailed above are carefully met, PPCCC with POBH is a very controlled and safe procedure which carries the potential of becoming a routine procedure. In any case, apart from pediatric cases, it may be considered in following clinical situations: pseudoexfoliation syndrome because of the pronounced tendency of capsular shrinkage; high myopia because of the significantly greater inclination to develop after-cataract; and peripheral retinal pathologies requiring controls and treatment (myopia, diabetes) because of the unimpeded insight for diagnosis and therapy.
Fig. 17 Repelling magnets located in the optic periphery (open square at 3/9 o'clock) and under the superior/inferior rectus muscle insertions. Upon ciliary muscle contraction/zonular relaxation, the optic is pushed forward, increasing the power of the IOL optic within the eye

\section{Accommodative IOL system}

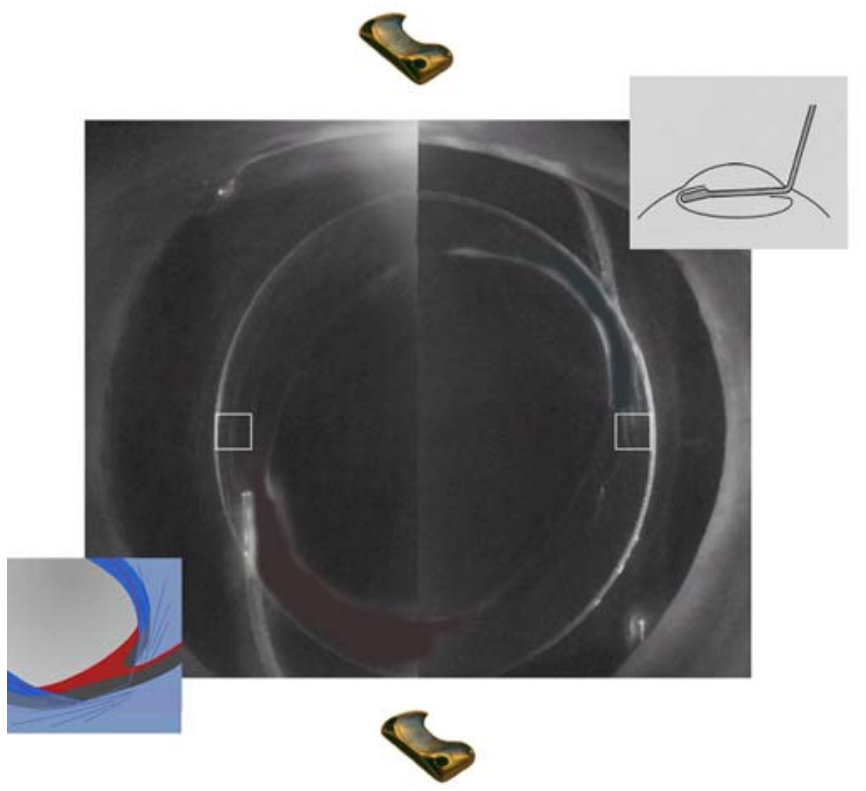


Two potential future applications may be mentioned separately:

1. Toric IOLs. Overly long plate and z-shaped open-loop haptics have been used to provide for rotational stability. However, these cannot completely avoid rotation and may interfere with capsular bag closure and thus after-cataract performance. Posterior rhexis fixation makes postoperative optic rotation impossible without the need for special haptics (Fig. 16).

2. Accommodative shift IOLs. Currently marketed passive-shift IOLs have been designed to move anteriorly within the capsular bag upon accommodative effort [14].

The fact is that any bag-fixated IOL is immobilized due to fibrotic shrinkage. Instead of moving the optic, movement of the whole IOL-capsule complex is therefore more promising. However, fibrotic distension of the zonules would again obviate axial movement. $\mathrm{POBH}$ drastically reduces capsular fibrosis, as the sandwiched posterior capsule precludes the contact between the anterior capsule and the optic as the prerequisite for LEC transdifferentiation. Additional polishing of the anterior capsule, with special regard to the areas adjacent to the haptic-optic junctions, has been shown to keep the capsule fully transparent and possibly elastic enough to allow axial movement of the optic under ciliary muscle contraction. This may fulfil the conditions of sufficient passive axial mobility, as postulated by Preussner, to allow for clinically relevant shift-induced accommodation when embedded in his magnet-driven concept [17] (Figure 17). Instead of implementing the magnets into a special CTR as suggested by Preussner, these could be directly mounted to the optic periphery. A study investigating passive mobility under pharmacological stimulation with cycloplegics and pilocarpine is ongoing.

Table 2 summarizes current and potential future indications.

In conclusion, primary posterior capsulorhexis combined with posterior optic buttonholing is a well-controlled, safe, and highly effective procedure with a steep learning curve, thus carrying the potential of becoming a routine alternative to standard in-the-bag implantation of intraocular lenses. As opposed to standard in-the-bag placement, its effectiveness is completely independent of optic material and optic edge design. This technique is meant for the skilled and dedicated surgeon, and must be carefully approached. It should be reserved to large pupil eyes. Should the pupil come down during the surgery, one may switch to standard in-the-bag surgery, optionally combined with a small PPCCC. Thorough viscodissection of anterior hyaloid and posterior capsule is crucial. Additional anterior capsule polishing is highly recommended, as it abolishes any residual fibrosis without any risks or downsides. Though long-term results in children's eyes support sustained efficacy and lack of complications, 5 -year results should be awaited until the technique may be considered or recommended as a routine alternative to standard in-the-bag fixation of the IOL. For the time being, it is recommended with pseudoexfoliation syndrome, high axial myopia, peripheral retinal disease, multifocal IOLs, and toric IOLs when made available.

Open Access This article is distributed under the terms of the Creative Commons Attribution Noncommercial License which permits any noncommercial use, distribution, and reproduction in any medium, provided the original author(s) and source are credited.

\section{References}

1. Abela-Formanek C, Amon M, Schild G, Schauersberger J, Heinze G, Kruger A (2002) Uveal and capsular biocompatibility of hydrophilic acrylic, hydrophobic acrylic, and silicone intraocular lenses. J Cataract Refract Surg 28:50-61

2. Davison JA (2004) Neodynium:YAG laser posterior capsulotomy after implantation of AcrySof intraocular lenses. J Cataract Refract Surg 30:1492-1500

3. Davison JA (2005) Consultation section. J Cataract Refract Surg 31:651-660

4. De Groot V, Hubert M, Van Best JA, Engelen S, Van Aelst S, Tassignon MJ (2003) Lack of fluorophotometric evidence of aqueous-vitreous barrier disruption after posterior capsulorhexis. $\mathrm{J}$ Cataract Refract Surg 29:2330-2338

5. Georgopoulos M, Menapace R, Findl O, Petternel V, Kiss B, Rainer G (2003) After-cataract in adults with primary posterior capsulorhexis: comparison of hydrogel and silicone intraocular lenses with round edges after 2 years. J Cataract Refract Surg 29:955-960

6. Gimbel HV (1997) Posterior continuous curvilinear capsulorhexis and optic capture of the intraocular lens to prevent secondary opacification in pediatric cataract surgery. J Cataract Refract Surg 23(Suppl 1):652-656

7. Grieshaber MC, Pienaar A, Stegmann R (2005) Posterior vertical capsulotomy with optic entrapment of the intraocular lens in congenital cataracts for prevention of capsular opacification. J Cataract Refract Surg 31:886-894

8. Kappelhof JP, Vrensen GF (1992) The pathology of after-cataract. A minireview. Acta Ophthalmol Suppl 205:13-24

9. Menapace R, Findl O, Georgopoulos M, Rainer G, Vass C, Schmetterer K (2000) The capsular tension ring: designs, applications, and techniques. J Cataract Refract Surg 26:898-912

10. Menapace R (2004) Prevention of posterior capsule opacification. In: Kohnen T, Koch DD (eds) Cataract and Refractive Surgery, Essentials in Ophthalmology. Springer New York, pp 101-122

11. Menapace R, Wirtitsch M, Findl O, Buehl W, Kriechbaum K, Sacu S (2005) Effect of anterior capsule polishing on posterior capsular opacification and Neodymium-YAG capsulotomy rate: a three-year randomized trial. J Cataract Refract Surg 31:20672075

12. Menapace R (2006) Routine posterior optic buttonholing for aftercataract eradication in adults: A report on 500 consecutive cases. J Cataract Refract Surg 32:929-943

13. Menapace R, Di Nardo S (2006) "Aspiration Curette": an instrument for efficient and safe anterior capsule polishing. Laboratory and clinical evaluation. J Cataract Refract Surg 32:1997-2003

14. Menapace R, Findl O, Kriechbaum K, Leydolt-Koeppl Ch (2007) Accommodating intraocular lenses: a critical review of present and future concepts. Graefes Arch Clin Exp Ophthalmol 245: 473-489 
15. Nishi O, Yamamoto N, Nishi K, Nishi Y (2007) Contact inhibition of migrating lens epithelial cells at the capsular bend created by a sharp-edged intraocular lens after cataract surgery. J Cataract Refract Surg 33:1065-1070

16. Olson RJ (2005) Consultation section. J Cataract Refract Surg 31:651-660

17. Preussner PR, Wahl J, Gerl R, Kreiner C (2001) [Accommodative lens implant]. Ophthalmologe 98:97-102

18. Ripandelli G, Coppe AM, Parisi V, Olzi D, Scassa C, Chiaravalloti A, Stirpe M (2007) Posterior vitreous detachment and retinal detachment after cataract surgery. Ophthalmology 114:692-697

19. Sacu S, Menapace R, Wirtitsch M, Buehl W, Rainer G, Findl O (2004) Effect of anterior capsule polishing on fibrotic capsule opacification: three-year results. J Cataract Refract Surg 30:2322-2327

20. Stifter E, Menapace R, Luksch A, Neumayer T, Vock L, Sacu S (2007) Objective assessment of intraocular flare after cataract surgery with combined primary posterior capsulorhexis and posterior optic buttonholing in adults. Brit J Ophthalmol 91:1481-1484

21. Stifter E, Luksch A, Menapace R (2007) Postoperative course of intraocular pressure after cataract surgery with combined primary posterior capsulorhexis and posterior optic buttonholing. J Cataract Refract Surg 33:1585-1590
22. Stifter E, Menapace R, Luksch A, Neumayer T, Sacu S (2008) Anterior chamber depth and change of axial intraocular lens position after cataract surgery with primary posterior capsulorhexis and posterior optic buttonholing. J Cataract Refract Surg (in press)

23. Stifter E, Luksch A, Menapace R (2008) Macular morphology and central retinal thickness after cataract surgery with primary posterior capsulorhexis and posterior optic buttonholing. Am J Ophthalmol (in press)

24. Sugita M, Kato S, Sugita G, Oshika T (2004) Migration of lens epithelial cells through haptic root of single-piece acrylic-foldable intraocular lens. Am J Ophthalmol 137:377-379

25. Tassignon MJ, De Groot V, Vervecken F, Van Tenten Y (1998) Secondary reclosure of posteriuor continuous curvilinear capsulorhexis in normal eyes and eyes at risk for postoperative inflammation. J Cataract Refract Surg 24:1333-1338

26. van Cauwenberge F, Rakic JM, Galand A (1997) Complicated posterior capsulorhexis: aetiology, management, and outcome. $\mathrm{Br}$ J Ophthalmol 81:195-198

27. Vock L, Cernej A, Findl O, Neumayer T, Buehl W, Sacu S, Rainer G, Menapace R. Effect of optic material on posterior capsule opacification in intraocular lenses with sharp-edge optics: 5 year results of a randomized trial. Ophthalmology (submitted) 